\title{
Necator americanus
}

National Cancer Institute

\section{Source}

National Cancer Institute. Necator americanus. NCI Thesaurus. Code C123524.

A species of parasitic nematodes in the family Ancylostomatidae. N. americanus can be passed person-to-person through contact with infested feces. 